\title{
ORGANISATIONS INTERNATIONALES, HUMANITAIRE ET LUSTRE CULTUREL
}

\section{Bernadette Dufrene ${ }^{1}$}

L'artiste Thomas Hirschhorn présentait lors de la biennale de Lyon Partages d'exotismes (2000) une cuvre intitulée United Nations-Miniature : l'installation, en forme de golf miniature, évoque un monde désolé où seuls subsistent et circulent les véhicules d'une blancheur immaculée avec l'inscription en lettres bleues UN (United Nations). Esthétique des organisations internationales dont l'ONU est le symbole : blanc/bleu, couleur de la paix, véhicules du temps, à la fois belles mécaniques et symboles d'aventure, prestige du logo, identifiable par tous les habitants de la planète.

L'objet de cet article n'est pourtant pas une interrogation sur la représentation des organisations internationales mais sur les liens que l'esthétique de ces organisations, -pour beaucoup issues des conflits qu'elles ont pour mission de prévenir-entretient avec le politique. Le point de départ de cette interrogation est double : il nous est fourni, d'une part, par l'analyse qui clôt le célèbre article de Walter Benjamin "L'œuvre d'art à l'ère de sa reproductibilité technique" (1936)2,

\footnotetext{
1 Maître de conférences à l'Université de Grenoble II.

2 W. BENJAMIN, "L'œuvre d'art à l'ère de sa reproductibilité technique" (1936), in Ecrits français, trad. Pierre Klossowski, Paris, Gallimard, 1991.
} 
d'autre part, par l'observation que l'on peut faire de l'utilisation et de la production de l'esthétique (au sens de ce qui affecte les sens et non la seule raison) dans les organisations internationales. Ce qui guide la réflexion, c'est le problème posé par Benjamin à propos de l'esthétisation du politique qui conduirait nécessairement à la guerre et à quoi il oppose une politisation de l'esthétique ${ }^{1}$. Les termes qu'emploie W. Benjamin "esthétisation", "politisation de l'esthétique" appellent un éclaircissement : le suffixe "-tion" qui désigne une action en cours invite à une analyse à la fois historique et pragmatique qui tienne compte de son cadre spécifique. Dans cet écrit de 1936, l'esthétisation a une connotation péjorative : le mot suggère dans ce contexte l'asservissement des moyens propres à l'esthétique, l'influence qu'elle exerce sur notre perception et notre sensibilité à des fins contraires aux valeurs humanistes. Au rebours, le texte de Benjamin laisse entendre que, si la "politisation de l'esthétique" voulue par le communisme a une connotation positive, c'est que le projet esthétique fait pleinement partie d'un projet social : dans les années vingt, les constructivistes rêvaient dans la nouvelle Union soviétique d'introduire l'art dans la vie. L'esthétique de W. Benjamin, loin d'être dissociable d'intérêts politiques immanents, leur est fortement corrélée : en cela elle s'oppose à une démarche transcendantale de type kantien qui la définirait par des conditions de possibilité a-historiques. Ancrée pour une part dans une problématique de l'action, elle pose la question de la responsabilité.

Reprendre la réflexion de Benjamin aujourd'hui en l'appliquant aux organisations internationales, c'est nécessairement la décontextualiser, -ce qui ne lui enlève pourtant pas sa pertinence-, même si l'esthétisation ne répond pas aux mêmes buts au sein de ces dispositifs voués au maintien de la paix et à la prévention des conflits. La question de l'esthétisation du politique pose celle de sa nature et de ses fonctions dans ce type d'organisations. Comment la caractériser dans ce cas? N'est-elle qu'un lustre culturel dont la figure la plus achevée serait l'humanitaire et qui masquerait des enjeux radicalement différents? Peut-on déceler des effets inattendus qui seraient une amorce de réalisation du souhait de Benjamin, une politisation de l'esthétique dont elles seraient les opérateurs?

1 Benjamin écrit : "Toutes les tentatives d'esthétisation de la politique ont le même point culminant. Ce point culminant est la guerre... Voilà ce qu'il en est de l'esthétisation de la politique que le fascisme encourage. Le communisme lui répond par la politisation de l'art". 
Pour avoir une compréhension des liens entre politique et esthétique, encore faut-il s'intéresser aux conditions d'émergence des grandes organisations internationales et à leur généalogie. Cela permettra, dans une perspective pragmatique, de dégager et d'analyser certaines formes d'esthétisation en fonction du contexte politique, de mettre en évidence les formes innovantes. Par ailleurs cette perspective devrait pouvoir permettre de rendre compte de la modification des enjeux : les organisations internationales peuvent-elles servir à une politisation de l'esthétique à l'heure de la mondialisation ?

\section{L'esthétisation de la médiation}

L'analyse de l'esthétisation d'une politique, du processus mis en œuvre par des organisations internationales demande que l'on dissocie au moins deux plans : l'esthétique des organisations internationales -produit de leur fonctionnement- et l'esthétique dans ces organisations, à savoir l'utilisation qu'elles font des formes d'expression artistique. Quand Bruno Ollivier"1 "met en relation le non-verbal organisationnel avec l'appartenance et la socialisation", il met l'accent sur la fonction esthétique "allant jusqu'à se confondre avec les processus de médiation institutionnelle", l'espace organisationnel et les systèmes d'objets symboliques mis en place ayant, selon lui, comme fonction principale "d'exhiber et de produire de l'appartenance en transmettant des représentations (du système, des acteurs, de leurs rôles et de leurs fins)". Ce point de vue sur l'esthétique des organisations peut être encore enrichi lorsqu'on le confronte à celui de W. Benjamin ${ }^{2}$ qui, en mettant en cause l'esthétisation de la politique à propos des grandes manifestations hitlériennes, souligne le mode d'action spécifique mis en cuvre: la dimension spectaculaire favorisant l'immersion de l'individu et l'abandon d'un point de vue critique. Mutatis mutandis, c'est d'abord à travers ces deux aspects que nous analyserons ce qui fait la spécificité de l'esthétique des organisations internationales : il s'agit à la fois de voir en quoi leur fonctionnement induit une

l B. Ollivier, "Non-verbal, médiation, stéréotypes : à côté des discours et de l'organisation ou en amont et en dessous ?", in H. HoTIER (dir.), Non-verbal et organisation, Paris, L'Harmattan, 2000.

2 A propos d'autres aspects de l'esthétique de Benjamin et notamment à propos de la métaphore de l'aura, voir la synthèse de Jean Caune dans Esthétique de la communication, Paris, PUF, 1997. 
esthétique qui leur est propre, comment ces organisations mettent en scène les postulats qui fondent leur action politique.

Par ailleurs on ne saurait envisager la question de l'esthétique de ces organisations sans nous interroger sur la place que tient l'art non seulement dans leurs murs mais aussi dans leur action. Toutes ces raisons rendent nécessaire de commencer par définir le type d'organisations sur lequel portera l'analyse et de rappeler les conditions de leur émergence.

\section{L'aura des organisations internationales}

C'est sans doute à l'écrivain Albert Cohen qu'il revient d'avoir évoqué, fût-ce sur le mode ironique, le prestige des organisations internationales : qu'on se souvienne de la fierté d'Adrien Deume, l'un des personnages de Belle du seigneur (1968), fonctionnaire à la SDN : "Arrivé devant le Palais des Nations, il le savoura. Levant la tête et aspirant fort par les narines, il en aima la puissance et les traitements. Un officiel, il était un officiel, nom d'un chien, et il travaillait dans un palais, un palais immense, tout neuf, archimoderne...".

Les organisations internationales nées des grands conflits du XX siècle et dont Armand Mattelart ${ }^{1}$ voit l'origine dans les sociétés de la

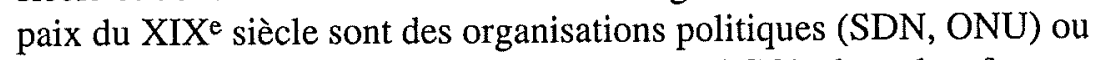
des services publics internationaux (UNESCO) dont les formes changent avec l'histoire; deux de ces changements sont particulièrement importants dans l'optique qui est la nôtre : si la SDN créée en 1919 n'admettait pas les colonies en son sein (les peuples colonisés sont représentés seulement par leurs tutelles), si, sur un autre plan, elle refuse de placer son action sur le terrain de l'éducation, en revanche, au lendemain de la seconde guerre mondiale, l'ONU non seulement admet une diversité de nouveaux États-nations mais de plus crée en novembre 1945 l'UNESCO (United Nations Educational, Scientific and Cultural Organization). Ces organismes supra-nationaux qui incarnent la puissance publique internationale ont surtout un rôle de médiation : ils ont en charge la paix et la sécurité. C'est d'ailleurs ce rôle de tiers symbolisant que les logos de l'ONU et de l'UNESCO (les

1 Armand Mattelart retrace l'apparition des associations internationales et de certaines organisations dans son ouvrage Histoire de l'utopie planétaire, Paris, La Découverte, 1999. 
rameaux autour du planisphère d'une part, la façade d'un temple grec d'autre part) font apparaître.

Ce rôle de tiers symbolisant puise naturellement dans l'idéologie de l'universalisme telle qu'elle se dégage de la Charte fondatrice des Nations Unies (1945) dont il suffit de rappeler quelques mots :

Nous, peuples des Nations Unies, résolus

à préserver les générations futures du fléau de la guerre

à proclamer à nouveau notre foi dans les droits fondamentaux de l'homme, dans la dignité et la valeur de la personne humaine, dans l'égalité des droits des hommes et des femmes, ainsi que des nations grandes et petites...

La Déclaration universelle des droits de l'homme (1948) sur laquelle l'organisation s'appuie fait référence à la Déclaration des droits de l'homme et du citoyen (1789). Même si le droit abstrait que celle-ci prône a été critiqué d'un point de vue historiciste dans la mesure où il s'impose comme transcendance aux sociétés humaines, sans tenir compte des déterminations dont elles sont le produit ${ }^{1}$, par leur seule force morale, néanmoins, en 1945 et en 1948, c'est cet universalisme reconnaissant à tout homme les mêmes droits et les mêmes devoirs qui légitime l'action de ces organisations et leur permettent d'assurer une médiation entre les États.

Daniel Bougnoux, s'appuyant à la fois sur Hegel et les travaux de Girard et Debray, précise la nature de la médiation symbolique : "Entrer dans le symbolique, c'est accepter la médiation d'un ordre émergent qui, n'étant institué par aucun partenaire particulier, peut les contenir tous"' ${ }^{2}$. C'est précisément le rôle que joue l'universalisme des Déclarations ; c'est ce qui donne l'aura des organisations internationales : dès la formulation de la Charte, éthique et esthétique de la médiation par le biais de l'universel ont partie liée. L'aura est une métaphore commode pour désigner les implicites du guidage et la manière dont nos sens sont affectés par l'évocation de ces organisations ; elle se prolonge par la mise en œuvre d'une esthétique propre aux organisations internationales.

1 Voir à ce sujet l'article de Luc Ferry et Alain Renaut, "Le fondement universel des droits de l'homme", Communication, ${ }^{\circ} 43,1986$.

2 D. Bougnoux, La communication par la bande, Paris, La Découverte, 1992. 


\section{Les Palais de la paix : une esthétique de l'universel et de la transparence}

Parmi les éléments qui constituent l'image des organisations internationales, l'architecture du siège est un élément clef non seulement dans la mesure où elle manifeste le prestige mais aussi dans celle où elle rend sensible la fonction. Si on a pu parler de "palais de la paix", c'est en raison de leurs dimensions monumentales qui sacralisent leur projet.

Le premier, le Palais des Nations, celui de la SDN à Genève -la Suisse, pays neutre, pays de Dunant et de la Croix-Rouge, siège du Bureau international des sociétés de la paix (Berne)-, a été réalisé entre 1929 et 1935 par les architectes Nenot et Flegenheimer. Deux blocs, l'un officiel et d'apparat, l'autre administratif, donnent dans le paysage genevois une impression de grandiose, de massif et d'élancé à la fois comme la montagne alpine toute proche. Équilibre classique et dépouillement moderne ${ }^{1}$ se conjuguent, comparables aux qualités dont témoigne le palais de Chaillot à Paris conçu à la même époque. L'immeuble des Nations Unies (1949-1953) à New York -à l'invitation des États-Unis en 1946, le centre des nations rassemblées fut transféré du Vieux Continent à l'Amérique- est né d'une idée de Le Corbusier : un gratte-ciel rectangulaire, puissant, entièrement vitré sur ses deux faces, les deux côtés étant laissés en marbre nu. A la rhétorique du décor du premier palais, fait place le brutalisme: une nouvelle façon de dire le droit par l'architecture. Architecture parlante, inhumaine si l'on considère les formes éloquentes et "humaines, trop humaines", pour paraphraser Nietzsche, du Palais de Genève. Quant au bâtiment de l'UNESCO derrière l'École Militaire à Paris, André Maurois le présentait comme un "palais de facture moderne"; "des architectes et des artistes de plusieurs pays ont participé à ses plans et à sa décoration"2. La photographie jointe montrait au premier plan la sculpture du britannique Henry Moore. Si la façade du Y qui forme le plan du bâtiment -symbole dynamique de la victoire de la paix- donnant sur l'École militaire, est sobre, en

1 L. Chéronnet, "Le Palais de la Société des Nations", Paris, L'Illustration, 1938.

2 A. Maurors, Paris, Paris, Nathan, 1969. 
revanche, le modernisme du porche relevé, des pilotis de béton voulus par les architectes Nervi, Zehrfuss et Breuer, éclate au centre du Y.

Mais si l'architecture d'ensemble conforte le prestige des organisations internationales, l'esthétique des "palais de la Paix" joue aussi sur l'idée d'universalité.

Ainsi le Palais des Nations à Genève comportait un somptueux décor peint par l'artiste espagnol José Maria Sert en 1935-1936, dans l'esprit de la grande iconographie baroque qui, autrefois, célébrait l'unité réalisée par l'Église. Le thème en est "ce qui sépare et ce qui unit les hommes"1. Dans la salle des conseils, de gigantesques grisailles traitent de sujets allégoriques (Justice, Force, Mort, Paix) Le plafond de la salle montre cinq colosses, qui symbolisent les cinq continents, debout sur des superstructures, tendant les bras pour joindre leurs mains au centre du plafond, composant une sorte de clé de voûte symbolique. Non moins intéressantes les scènes qui sont figurées en dessous d'eux : on y voit l'université de Salamanque telle qu'au XV'e siècle le juriste Francisco Vitoria y posa les bases du droit international. On appelle cette composition La solidarité des peuples ou Leçon de Salamanque. Ailleurs, ce sont les Progrès de la science et les Progrès techniques, l'accent étant mis sur le chemin de fer qui relie les hommes. Le décor est celui de la grande Église humaine, éthique fondée sur une foi dans le progrès ${ }^{2}$ : Palais de la foi laïque, décor destiné à rassembler. Mais le Musée des Arts Océaniens et Africains, ex-Palais des Colonies (1931), rappelle que l'on rassemble souvent pour hiérarchiser et que l'on rassemble en image les sujets des quatre coins du monde au lieu qui les commande.

L'architecture de l'immeuble des Nations Unies à New York n'est pas moins parlante : la coupole de l'auditorium du bâtiment de l'Assemblée générale retrouve symboliquement la forme sphérique de l'unité. C'est aussi cette esthétique de la médiation que l'on trouve dans 1'œuvre du Chinois Chen Zhen Round Table (1995) placée quelque temps devant le bâtiment: des chaises venues des cinq continents sont disposées autour d'une table ronde en bois dont le centre comporte un extrait de la Déclaration des droits de l'Homme écrit en caractères chinois. Par ailleurs, l'immensité des baies vitrées

\footnotetext{
1 José Maria Sert, 13 septembre 1934, cité par Arturo Colorado Castellary, Les peintures murales de José Maria Sert, New York, Nations-Unies, 1985, p. 11.

2 Cf. P. Claudel, "Une visite au Palais de la Société des Nations", in L'ceil écoute, Paris, Gallimard, 1960.
} 
donne l'impression de la transparence dans une ruche, celle de l'activité des délégués et des fonctionnaires.

Si l'architecture et les éléments de décor qui s'y rattachent rendent manifeste l'éthique de l'organisation, c'est qu'ils sont conçus comme partie intégrante de l'image de l'organisation. Sacralisée par la réalisation de bâtiments prestigieux, l'éthique de la médiation des organisations internationales est aussi rendue visible.

La visibilité de cette éthique s'appuie aussi sur une autre forme de communication tridimensionnelle : l'exposition.

\section{La logique d'exposition dans les organisations internationales}

Le siège des organisations internationales est par excellence un lieu d'exposition, que l'exposition soit permanente ou temporaire : une exposition permanente de sculptures et des expositions temporaires célèbrent la vocation culturelle de l'UNESCO tandis que le bâtiment de l'ONU accueille les "trésors" de l'humanité.

On peut se demander pourquoi, alors même que les sociétés de la paix qui décidèrent, lors d'une réunion à l'occasion de l'Exposition universelle de 1889 , de tenir un congrès annuel, n'avaient eu pour seul souci que de réunir une "bibliothèque universelle", les œuvres d'art sont venues se joindre à ce qu' on n' appelait pas encore la "liste du patrimoine de l'humanité" dans l'idée de l'organisation internationale.

Un fait rapporté par A. Mattelart ${ }^{1}$ peut conduire à un début de réponse. Il s'agit de la déclaration faite justement en 1889 à Paris par le pacifiste Frédéric Passy à Paris devant les organisations pacifistes : "Où pourrait-on prononcer plus justement ces paroles que dans le lieu où nous sommes? Où en trouve-t-on la démonstration plus évidente qu'au milieu de toutes ces merveilles du travail du genre humain tout entier qui sont amoncelées à quelques pas d'ici (...) Ce que nous enseignent là-bas les choses qui sont exposées sous nos yeux, la fraternité du genre humain, nous venons ici de tous les coins du monde la proclamer, (...) je dis que nous avons ici une sorte de représentation morale de l'espèce humaine".

1 A. MATTELART, op.cit. pp. 200-201. Nous soulignons. 
Passy renoue sous les auspices de la question de la paix universelle avec le motif de la liste des merveilles de l'humanité élaborée dès l'Antiquité. Ces merveilles, le phare d'Alexandrie, les jardins suspendus de Babylone conjuguaient l'invention technique et le génie artistique. La nouveauté est ici de voir dans la "co-présence" des inventions techniques et scientifiques et des cuvres d'art de tous les pays le lien entre l'esthétique ("représentation") et l'éthique ("morale").

Dans Belle du Seigneur, déjà cité, Deume fait faire une visite du Palais des Nations à sa femme'. Pour l'épater, "il mentionna fièrement les dons des divers pays : les tapis de la Perse, les bois de la Norvège, les tapisseries de la France (des Gobelins), les marbres de l'Italie, les peintures de l'Espagne et toutes les autres offrandes...". Aujourd'hui, c'est à New York que se donne à voir le trésor des Nations, que des guides zélés, modernes Deume, présentent avec la même fierté dans toutes les langues: tapisseries, ivoires, symboles en tout genre de l'unité (barques, lampes, cloche de la Paix offerte par le Japon), œuvres d'art. Le jardin de sculptures qui donne sur l'East River se signale par des monuments à la paix offerts par les pays de l'Est. L'exposition des trésors de l'humanité a, dans ce contexte, un sens très différent de celui qu'il aurait dans un musée ou une exposition : au nom de l'universel, les organisations internationales opèrent une légitimation de la diversité culturelle. En cela, leur propos n'est pas éloigné de celui des Expositions universelles, du moins sous leur forme contemporaine, ou d'expositions comme Magiciens de la terre (Paris, 1989) dont le but était de faire l'inventaire de la création artistique dans le monde entier et non dans sa seule composante occidentale.

Si la légitimation de la diversité culturelle est rendue possible par l'exposition des trésors de l'humanité, c'est qu'elle s'appuie sur la puissance d'un postulat : l'art comme langage universel qui peut être compris de tous. André Malraux, animé par l'idée du Musée imaginaire, proclame en 1960, à l'occasion du lancement de la campagne de sauvegarde des monuments de Nubie sous l'égide de l'UNESCO, que toutes les œuvres constituent le "trésor de la première civilisation mondiale" :

1 A. CoHEN, Belle du seigneur, Paris, Gallimard, 1968, pp. 58-59. 
Pour la première fois, l'humanité a découvert un langage universel de l'art. Nous en éprouvons clairement la force, bien que nous en connaissions mal la nature. Sans doute cette force tient-elle à ce que ce Trésor de l'Art dont l'humanité prend conscience pour la première fois, nous apporte la plus éclatante victoire des œuvres humaines sur la mort. A l'invincible "jamais plus" qui règne sur ces civilisations, ce trésor survivant oppose sa grandiose énigme ${ }^{1}$.

La conception de l'art comme langage universel fonde la possibilité de l'idée de patrimoine mondial ; dans le contexte des organisations internationales, l'exposition des trésors de l'humanité a un sens aussi éthique : le statut des œuvres n'est pas seulement celui d'attributs du pouvoir, d'éléments de prestige, il est aussi celui de témoins d'un patrimoine mondial revendiqué par la communauté internationale. Sur ce point, la conclusion de Malraux est éclairante :

au moment où elle rassemble les œuvres fraternelles de tant de civilisations qui se haïssent ou qui s'ignorent, la première civilisation mondiale revendique publiquement l'art mondial comme son indivisible héritage.

Ces propos permettent de comprendre que l'exposition des trésors de l'humanité au sein des organisations internationales est une partie constitutive de leur identité et de leur image comme tiers symbolisant.

Si l'éthique des organisations internationales induit une forme spécifique d'esthétique, ce que nous avons appelé l'esthétisation de la médiation, si elle constitue le fondement de leur image, est-ce que précisément cette image d'une médiation universaliste n'agit pas aussi comme un masque?

\section{L'esthétisation de la médiation comme masque}

L'esthétique de la médiation peut-elle jouer comme un leurre et rencontrer des résistances? Quel universalisme les organisations internationales promeuvent-elles ? A ce stade, il est utile d'avoir recours à la distinction que Maurice Merleau-Ponty établissait entre un "universel de surplomb d'une méthode strictement objective" et un

\footnotetext{
1 A. Malraux, "Adresse à l'UNESCO du 8 mars 1960".
} 
"universel latéral" défini comme "une incessante mise à l'épreuve de soi par l'autre et de l'autre par soi"'. De fait, ces organisations qui se veulent des instances de régulation au-delà des conflits politiques, économiques et au-delà des revendications identitaires ont-elles pu trouver dans l'esthétisation de la médiation un dépassement du réel ? L' "universel de surplomb" qui s'énonce dans des dispositifs esthétiques n'est-il pas plutôt de l'ordre de l'idéal ?

\section{L'esthétisation de la médiation comme masque du pouvoir}

Si l'éthique des organisations internationales est fondée sur le principe de la représentation de toutes les nations et de leur égalité, il n'en demeure pas moins qu'un certain nombre de filtres ont été mis en place : que l'on songe, par exemple, que le poids du vote du Conseil de sécurité de l'ONU est supérieur à celui de tous les autres membres. Le récent sommet du G8 a bien montré à quel point la co-présence pouvait être un leurre : il ne suffit pas d'inviter les chefs d'État des pays moins riches pour infléchir les orientations de l'économie même s'il s'agit là d'un début de reconnaissance. On est alors amené à se demander si l'esthétisation de la médiation ne masque pas un universalisme de surplomb. La sphère de l'auditorium qui, au siège de l'ONU, manifeste l'unité des Nations, ne dfissimule-t-elle pas l'inégalité entre les membres du Conseil de sécurité et les autres participants ? La fonction de cette esthétique architecturale pourrait être alors rapprochée de celle de la courtoisie dans les conférences diplomatiques : préserver et respecter les formes pour entretenir un lien. Les logos de l'ONU et de l'UNESCO peuvent aussi servir de base de réflexion : au moment même où c'est le consensus des nations qui est recherché, ce sont des symboles propres au monde occidental qui sont utilisés : les rameaux pour l'ONU, la façade de temple grec pour l'UNESCO.

La logique de restauration du patrimoine à l'œuvre dans les zones de conflit peut aussi avoir valeur de démonstration de force. La communauté internationale impose sa volonté et sa puissance politique : la reconstruction du pont de Mostar et de la bibliothèque austro-hongroise de Sarajevo en constituent un symbole spectaculaire. $\mathrm{Ne}$ voit-on pas dans ces deux cas exemplaires la mise en pratique de

1 M. Merleau-Ponty, Signes, Paris, Gallimard, 1960. 
ce qui sert d'emblème au Patrimoine mondial : un carré noir sur la pointe lié par deux traits partant de l'angle inférieur à un cercle qui l'entoure ? Ainsi le bâtiment digne d'intérêt est protégé par le cercle des nations qui l'aident à renaître.

\section{Esthétisation de la médiation/politisation de l'esthétique : la difficulté du passage}

L'esthétisation de la médiation, l'universalisme affiché par les organisations internationales, masquent aussi les difficultés auxquelles se heurte une médiation internationale en matière culturelle. Une politisation de l'esthétique suppose une politique culturelle, c'est-à-dire un ensemble de moyens mis au service des principes fondateurs des organisations internationales, de leurs objectifs. Elle suppose plus qu'une tactique, une stratégie ; la difficulté du passage entre la création de formes symboliques et leur utilisation à une realpolitik pose à la fois un problème de communication interculturelle et un problème de communication institutionnelle. André Chastel, dans un éditorial de la Revue de l'Art de 1977', dénonce deux obstacles à l'établissement d'une politique : les nationalismes et la tentation du spectaculaire :

En 1945 -il y avait bien peu de temps qu'un Romain Rolland était mort- on pouvait encore penser que l'art serait reconnu comme un langage universel, comme le langage universel. C'était ne pas compter avec la flambée des nationalismes, phénomène inattendu, mais capital de l'après-guerre.

Et d'évoquer les demandes de restitution d'œuvres d'art (Grèce) ou ceux qui affirmaient "que mettre en valeur et diffuser l'œuvre de Michel-Ange ou de Rembrandt constituait un attentat envers les cultures autochtones, et tout compte fait, procédait d'une forme détournée, mais d'autant plus pernicieuse, de "néo-colonialisme". Faute de pouvoir ou de vouloir affronter des nationalismes, "la tentation sera grande de préférer des champs d'action où le consensus est plus facile, ou de se replier sur des manifestations louables, mais bénignes". Pour A. Chastel, l'action de l'UNESCO était

1 Revue de l'Art, $\mathrm{n}^{\circ} 37,1977$. Repris dans "Les organisations internationales", in Chastel présente la Revue de l'art (éditoriaux), Paris, Flammarion, 1980, pp. 2532. 
essentiellement déclinée en "quelques interventions spectaculaires, comme la reconstruction des temples de Philae, (...) les comités pour le salut de Venise ou de l'Acropole" auxquelles forcément le grand public est sensible.

Vingt ans auparavant, Malraux exaltait tout au contraire ces interventions et mettait en parallèle sans les opposer la sauvegarde du patrimoine artistique et l'humanitaire :

Pour le patrimoine artistique des hommes, vous faites appel à la conscience universelle comme d'autres le font cette semaine pour les victimes de la catastrophe d'Agadir. "Puissions-nous n'avoir pas à choisir, avez-vous dit tout à l'heure, entre les effigies de porphyre et les vivants !". Pour la première fois, vous proposez de mettre au service des effigies, pour les sauver, les immenses moyens que l'on avait mis, jusqu'ici, au service des vivants.

Le passage d'une esthétisation de la médiation par des actions spectaculaires à une véritable politique se heurte à ce qu'on pourrait nommer "le retour du refoulé" : la revendication culturelle nationaliste qui entre en conflit avec l'idée même d'un patrimoine mondial.

\section{Mémoire et conflits d'échelle}

Outre les nationalismes culturels, la question de la mémoire sur laquelle repose l'inscription d'un site comme patrimoine de l'humanité est une raison de la difficulté du passage à une politique de l'esthétique au niveau international. "L'universel de surplomb" que véhiculent les organisations internationales s'accorde mal avec la prérogative des États-nations en matière de patrimoine. Les avantages de l'inscription sur la Liste du Patrimoine mondial sont pourtant nombreux; Léon Pressouyre souligne fortement les effets bénéfiques d'une telle inscription : effet moral avec la reconnaissance internationale et le prestige, effet économique, avec l'augmentation du budget et ses retombées, effet d'entraînement enfin en faveur d'autres sites non encore inscrits ${ }^{1}$. Le fait que des États s'opposent à cette médiation internationale montre les limites d'une esthétique universaliste quand elle se heurte à des enjeux historiques ou politiques. Deux exemples

1 Voir L. Pressouyre La Convention du Patrimoine mondial, 20 ans après, UNESCO, 1993. 
en témoigneront : celui du Krak des Chevaliers en Syrie et celui des Bouddhas d'Afghanistan.

Le Krak des Chevaliers est un des exemples les mieux conservés au monde d'architecture fortifiée médiévale. La Syrie a toujours refusé de céder aux sollicitations de proposer cet édifice qui se trouve sur son sol au classement sur la Liste. L'argument est compréhensible : il s'agit d'un vestige des Croisades. La question posée est la suivante : faut-il, parce qu'un édifice est esthétiquement intéressant, un jalon ou un témoin de l'histoire de l'art, en oublier sa fonction politique et militaire ? Ou plutôt faut-il imposer à un pays d'adopter le seul point de vue artistique ne le condamnerait-il pas, sous la contrainte morale et économique, à renoncer à gérer sa propre mémoire ? Le point de vue historiciste (on garde parce que cela a été) doit-il prévaloir sur le point de vue mémoriel (on signale ce dont on veut bien se souvenir) ? Pour les organisations internationales, le point de vue historiciste l'emporte. Pour la Syrie, il suffit d'entretenir le château. On rejoint la constatation de Pierre Nora: "On est passé... d'un patrimoine hérité à un patrimoine revendiqué"1. S'il n'est pas "revendiqué", il ne peut être proposé à l'inscription sur la Liste du Patrimoine mondial.

Le problème des Bouddhas d'Afghanistan est d'une autre nature puisque les Talibans n'ont accepté aucune solution de compromis proposée par l'UNESCO (masquage partiel ou total) et ont finalement détruit ces colosses issus d'une religion qui n'est pas la leur et d'une histoire dans laquelle ils ne se reconnaissent pas. La pression exercée sur eux à travers la question du patrimoine artistique révèle une autre fonction de la médiation à la fois esthétique et éthique exercée par les organisations internationales : rappeler les États à leur devoir d'ouverture au reste de l'humanité, à la discussion et à la tolérance.

Aussi, même si l'esthétisation de la médiation des organisations internationales agit comme masque des inégalités et montre surtout les limites d'un universalisme de surplomb, on ne peut que souligner en quoi elle est nécessaire à la communication internationale : en "habillant" les positions de "surplomb" (des grandes puissances, de la communauté internationale) par une mise en scène appropriée, elle exprime un consensus international notamment à l'encontre des

1 P. NORA, "Avant-propos", in M.-A. SIRE, La France du patrimoine : les choix de la mémoire, Paris, Gallimard, 1998. 
totalitarismes. Cependant elle révèle aussi la difficulté d'une politisation de l'esthétique qui articulerait échelles internationale et nationale. Celle-ci est-elle pour autant impossible?

\section{Voies d'une politisation de l'esthétique}

A quelles conditions peut-on considérer l'action des organisations internationales comme une politisation de l'esthétique? A quelles conditions sert-elle une vision à long terme et ne se limite-telle pas au caractère souvent spectaculaire de la culture ? La mise en question d'une forme d'esthétique événementielle nous amènera à tenter de définir d'autres voies de diffusion de l'esthétique.

\section{L'événementiel en question}

Pour E. Hall', une des difficultés les plus grandes de la communication interculturelle est ce qu'il appelle la culture cachée. Dans la même perspective, Jacques Demorgon insiste sur la nécessité de l'interculturel-source, c'est-à-dire la connaissance du contexte dans lequel se situe une action ${ }^{2}$. Sans cet ancrage, l'action des organisations internationales dans le domaine culturel comme dans d'autres domaines est vouée à la superficialité ; elle constituerait au mieux un secours d'urgence aux œuvres sans réelle implication locale, l'humanitaire culturel dont Malraux faisait l'apanage de la première civilisation mondiale. Apportée de l'extérieur, sans liens forts avec le contexte local, la culture dans ce cas est plutôt un lustre culturel ; nous avons déjà mentionné la dénonciation qu'A. Chastel faisait des grandes campagnes de sauvetage spectaculaires et consensuelles menées sous l'égide de l'UNESCO. A cela s'ajoutent les ratages événementiels : bien que le cas qui a défrayé la chronique à Sarajevo en 1997 -le don par l'Italie à Sarajevo d'une sculpture intitulée L'uomo multiculturale costruira il mondo (L'homme multiculturel construira le monde)- n'implique pas les organisations internationales, il est néanmoins éclairant de l'utilisation de l'esthétique comme

1 E. HaLl, Au-delà de la culture, Paris, Éd. du Seuil, 1979 (1976).

2 J. Demorgon, Complexité des cultures et de l'interculturel, Paris, Anthropos, 1996. 
lustre culturel dans les relations internationales. La statue d'un sculpteur italien, inaugurée en grande pompe la même année sur la place de la Libération à Sarajevo, apparut un matin avec un chiffon noué autour des hanches. La sculpture, L'homme multiculturel est un homme de bronze nu au milieu d'une sphère constituée de barres métalliques qui figurent les méridiens. Il se libère des méridiens qu'il tord pour devenir un "homme du monde" et de ce fait ouvre la cage à des oiseaux de bronze. Mais les meilleures intentions du monde ne font ni du bon art ni tout simplement de l'art respectueux. Pour l'association de musulmans bosniaques qui a revendiqué l'action, "cette culture n'appartient pas aux gens qui vivent dans nos contrées"1. Cette affaire montre sur quels thèmes l'approche critique peut appuyer sa dénonciation : inadéquation au contexte, événementialisation et spectacularisation de la culture (cérémonie du don pour marquer la fin de la guerre et l'avènement d'un multiculturel autoproclamé).

\section{L'apparition d'une nouvelle convention}

La présence des organisations internationales crée sur le terrain de nouvelles situations. Ces dernières sont le produit d'une histoire à la fois diachronique (souvent marquée par des conflits) et synchronique (les rapports à une époque donnée). La théorie d'Howard Becker ${ }^{2}$ centrée sur les conditions d'élaboration des conventions permet d'appréhender les changements et les innovations et d'appréhender de manière concrète l'action des organisations. Bien qu'il ne soit pas possible de les décrire ici dans leur complexité, on peut souligner un trait de leur fonctionnement qui peut s'apparenter à celui des multinationales, dans la mesure où s'y manifeste aussi le mot d'ordre : think globaly /act localy. Il induit une diffusion de normes et de pratiques à l'échelle internationale mais adaptée localement. L'écrivain Ivo Andric avait déjà souligné la puissance intégratrice de la culture diffusée par les établissements d'éducation autrichiens pour assimiler les Bosniaques. De la même façon, A. Chastel remarque qu'en fait, c'est par les ONG qu'elle peut s'affilier que l'“UNESCO

1 Cf. I. Lovrenovic, "Un cadeau multiculturel... et empoisonné", Feral Tribune (Split) repris dans Courrier international, $\mathrm{n}^{\circ} 417,29$ oct-4 novembre 1998, p. 21.

2 H. BECKER, Les Mondes de l'art, Paris, Flammarion, 1988 (1982). 
touche plus directement aux problèmes de l'art". Ainsi du Conseil international des Musées (Paris, 1946, ICOM) qui a effectué un travail de normalisation en matière de muséologie ; le CIMAM, son organisme pour les musées d'art moderne, réfléchit régulièrement dans ses congrès à la question des rapports entre art et nationalité, constituant une manière d'entraide et de laboratoire internationaux dans ce domaine. Dans la même optique, Chastel rapporte une initiative de l'UNESCO visant -à travers la formation- la diffusion de normes internationales conçues à l'échelle internationale : la frappe d'une médaille à l'occasion du quatre centième anniversaire de Rubens, dont la vente servit à former des étudiants d'art de pays en voie de développement.

Au sein de cette politisation de l'esthétique que constitue la diffusion mais aussi la négociation de normes internationales, un exemple d'“universalisme latéral" au sens de mise à l'épreuve de soi par l'autre et de l'autre par soi est fourni par l'étude menée actuellement par l'UNESCO en vue du classement d'un site bosnien. La revue Courrier international ${ }^{1}$ a publié un article éclairant paru dans la presse de Sarajevo. Colin Kaiser, le représentant de l'UNESCO, cité dans l'article, fait l'historique de la question et explique pourquoi la précédente candidature du Bascarsi (la ville turque de Sarajevo) n'a pas été retenue dans les années 1980 : à l'échelle de la planète, nécessairement comparative, où se situent les experts internationaux, "la qualité des édifices du Bascarsi a été jugée inférieure à celle de l'héritage ottoman d'Istanbul". Si le classement du fameux pont de Mostar (XVIe siècle), pratiquement reconstruit aujourd'hui, semble acquis, en ce qui concerne Sarajevo, la proposition est maintenant d'élargir le périmètre du classement :

Elle englobe la partie de la ville construite par l'Autriche. Cela rend Sarajevo très intéressant. On pénètre dans une ville qui ressemble à une ville européenne et l'on découvre que celle-ci cohabite avec la partie datant de la période ottomane.

Ainsi, la politisation de l'esthétique (reconnaître et faire reconnaître par ce classement le caractère multiculturel de la cité) a un caractère à la fois constatif et performatif : le projet fédérateur qui a été choisi, unit soit sous la forme d'un pont (on sait qu'Ivo Andric a

1 S. SEHERCEHAJiC, "Sarajevo et Mostar prochainement inscrits au patrimoine mondial", Courrier international ${ }^{\circ} 427,7-13$ janvier 1999, p. 18. 
fait du "pont sur la Drina" à Visegrad un véritable mythe, que même Peter Handke a cité comme exemple de "mémoire commune"1), soit sous la forme d'une aire urbaine regroupant deux périodes, et même plus que cela, une manière de "pont" entre l'Europe et l'Orient -dont les Bosniens sont fiers car l'on passe d'un quartier à l'autre, sans aucune césure, ni obstacle, comme dans un rêve et d'un seul coup, l'ambiance se modifie, quoiqu'on garde du quartier d'où l'on vient une impression diffuse qui à son tour se mêle aux couleurs et aux sons du quartier où l'on pénètre. Les Situationnistes, tenants de la psychogéographie et qui voulaient s'emparer du siège de 1'UNESCO', trouveraient à Sarajevo matière à une "dérive urbaine", base d'une géographie urbaine sensible et un sujet de réflexion : l'UNESCO, avec son objectif "global", peut reconnaître une situation locale. La question de la patrimonialisation, élément essentiel d'une politisation de l'esthétique, peut soulever une autre interrogation : dans son souci de sanctuariser des aires historiques, et de faire la chasse aux nouvelles constructions (considérées comme parasites ou "sauvages"), l'UNESCO ne s'enferme-t-elle pas paradoxalement dans l'esthétique puriste à laquelle elle semble tourner le dos en classant des zones historiquement hétérogènes?

L'exemple de cette action montre que, si la voie d'une politisation de l'esthétique est étroite, il n'en demeure pas moins qu'elle est pratiquée ; elle peut être définie comme un universalisme latéral.

"Si c'était à refaire, je commencerais par l'éducation et la culture", disait Jean Monnet à propos de l'Union européenne. Plus que l'économie, c'est la culture qui unit, ou qui divise. Loin d'être la superstructure, elle est la nervure, ou, si l'on veut se souvenir de la proposition de retournement de tout intérieur vers sa surface de contact faite récemment par François Dagognet, elle est la surface essentielle. Les "palais de la paix" ont été la nervure ou la colonne vertébrale. Dans les années 1960, des critiques de plus en plus nombreuses leur reprochaient leur forme concentrée : d'une part, du fait du lieu de leur implantation dans un bâtiment d'une capitale du monde occidental dont elles épouseraient le point de vue et, d'autre part, du fait de l'aspect de vastes opérations spectaculaires que

\footnotetext{
I P. HANDKE, Un voyage hivernal vers le Danube, la Save, la Morava et la Drina, trad. par Georges Lorfèvre, Paris, Gallimard, 1996.

2 Cf. A. MATtelarT, op. cit., p. 304.
} 
prenaient leurs actions. Si l'on se réfère à la question posée par W. Benjamin, celle de l'instrumentalisation de l'esthétique par le politique, on parlera alors d' "esthétisation" de la politique quand éthique et esthétique sont si fortement corrélées qu'elles ne peuvent que manifester l'identité de l'organisation, la nature de son pouvoir même si cette esthétisation a parfois, dans le cas que nous avons étudié, une fonction de masque des positions d'un universalisme de surplomb et peut faire l'objet de rejets ; on parlera de la "politisation de l'esthétique" quand l'organisation, dépassant l'événementiel, définit une stratégie en matière de culture, avec comme objectifs la diffusion de ses normes et leur adaptation locale; la voie vers un universalisme latéral est ouverte quand les recentrements imposés par des situations locales exigent de plus en plus d'études approfondies, de dialogue. Il faut voir peut-être un signe de ce que l'esthétique des organisations internationales est en train de se modifier dans leur capacité à accompagner des initiatives : l'esthétique de la médiation quand elle emprunte la voie de l'universalisme latéral ne peut être confondue avec celle de la concentration des multinationales. 